New York, New York 10032, USA. Phone: (212) 305-1665; Fax: (212) 305-3986; E-mail: eas3@columbia.edu.

1. DiMauro, S., and Schon, E.A. 2003. Mitochondrial respiratory-chain diseases. N. Engl. J. Med. 348:2656-2668.

2. Janssen, R.J., Van Den Heuvel, L.P., and Smei- tink, J.A. 2004. Genetic defects in the oxidative phosphorylation (OXPHOS) system. Expert Rev. Mol. Diagn. 4:143-156.

3. Zhu, Z., et al. 1998. SURF1, encoding a factor involved in the biogenesis of cytochrome $c$ oxidase, is mutated in Leigh syndrome. Nat. Genet. 20:337-343.

4. Tiranti, V., et al. 1998. Mutations of SURF-1 in Leigh disease associated with cytochrome c oxidase deficiency. Am. J. Hum. Genet. 63:1609-1621.

5. Mootha, V.K., et al. 2003. Identification of a gene causing human cytochrome $c$ oxidase deficiency by integrative genomics. Proc. Natl. Acad. Sci. U. S. A. 100:605-610.

6. Kirby, D.M., et al. 2004. NDUFS6 mutations are a novel cause of lethal neonatal mitochondrial complex I deficiency. J. Clin. Invest. 114:837-845. doi:10.1172/JCI200420683.

\title{
The link between IFN- $\gamma$ and allograft arteriopathy: is the answer NO?
}

\author{
Richard N. Mitchell and Andrew H. Lichtman
}

Center for Excellence in Vascular Biology, Department of Pathology, Brigham and Women's Hospital, Boston, Massachusetts, USA.

\begin{abstract}
Vascularized organ transplants often fail because of smooth muscle cell migration and proliferation in the intima of graft arteries, leading to progressive lumenal narrowing and resultant ischemic damage. Graft arterial disease is caused by IFN- $\gamma$ secreted by alloreactive T cells. New evidence indicates that IFN- $\gamma$ dysregulates expression of the enzymes eNOS and iNOS in graft-infiltrating leukocytes (see the related article beginning on page 846). Dysregulated NO synthase expression occurs prior to and is causally linked to intimal smooth muscle cell accumulation.
\end{abstract}

Graft arteriosclerosis is the major cause of late allograft failure. Pathologic features include arterial intimal hyperplasia due to recruitment and proliferation of smooth muscle cells (SMCs), which eventually causes lumenal obstruction and allograft ischemia (Figure 1, A and B). Although the mechanisms underlying graft arteriosclerosis are not well understood, the condition most likely results from a form of ongoing immune rejection, and most evidence suggests critical dependence on host $\mathrm{T}$ cell alloantigen recognition (1). Nevertheless, graft arteriosclerosis is resistant to standard immunosuppressive therapies. Of a plethora of candidate effector molecules, IFN- $\gamma$ - a proinflammatory cytokine produced by effector $\mathrm{T}$ cells has long been suspected of being a major player in graft arteriosclerosis.

\section{IFN- $\gamma$ causes graft arterial disease}

To dissect the molecular mechanisms underlying this process, in vivo models that recapitulate the changes observed following human organ transplants have

Nonstandard abbreviations used: EC, endothelial cell; SMC, smooth muscle cell.

Conflict of interest: The authors have declared that no conflict of interest exists.

Citation for this article: $J$. Clin. Invest. 114:762-764 (2004). doi:10.1172/JCI200422927. been developed; indeed, murine cardiac allografts develop arterial changes pathologically identical to the human disease. Using this model, arteriopathy does not develop in allografts transplanted into IFN- $\gamma$-deficient hosts or after treatment with anti-IFN- $\gamma$; this finding provided the first direct evidence that IFN- $\gamma$ is necessary to induce graft arteriosclerosis (2-4). However, the relevance of findings in a mouse model to human arteriopathy must be weighed in light of known interspecies differences in vascular cell phenotype and function, including those in endothelial cells (ECs) and SMCs (5).

Jordan Pober and colleagues previously addressed the issue of species differences using a model of graft arterial disease involving human arterial segments interposed in the infrarenal aorta of lymphocyte and NK cell-deficient (SCID/beige) mice (6-8). These arterial xenografts remain histologically normal in the immunodeficient host mice. However, if human IFN- $\gamma$ is administered, intimal thickening occurs as a result of SMC accumulation. Because the host mice are immunodeficient and murine cytokines do not have biological activity on human cells, the results indicate that IFN- $\gamma$ is also sufficient to induce graft arterial disease. Moreover, human arterial grafts in SCID/beige hosts injected with allogeneic
PBMCs also develop arteriopathy, associated with human IFN- $\gamma$-expressing T cell infiltrates. Administering anti-IFN- $\gamma$ reduces the intimal thickening caused by PBMC injection (6-8).

\section{NO and arterial dysfunction}

Although the data from murine allograft and xenograft models clearly establishes a central role for IFN- $\gamma$ in graft arterial disease, the pathologic mechanisms downstream of IFN- $\gamma$ are not clear. One possibility is that IFN- $\gamma$ dysregulates vascular wall production of and response to NO $(9,10)$. ECs synthesize NO from L-arginine via constitutively expressed eNOS; leukocytes can synthesize NO through iNOS. Induction of vasodilation (through vascular SMC relaxation) is one of the principal roles of NO synthesized by ECs via eNOS. However, NO does have other activities related to vascular wall function including inhibiting SMC proliferation and reducing platelet and leukocyte adhesion (11).

Dysregulated eNOS production - reflecting a more global EC dysfunction - can be assessed by measuring the vasomotor responsiveness of arteries to eNOS-dependent vasodilators. EC dysfunction is implicated in atherosclerosis and graft arteriosclerosis. In addition, failure of SMCs to relax in response to $\mathrm{NO}$ (SMC dysfunction) is also implicated in graft arteriosclerosis. Since both IFN- $\gamma$ and dysregulation of NO responses are associated with graft arterial disease, a logical question to ask is whether IFN- $\gamma$ is the cause of EC and vascular SMC dysfunction. In a report by Pober, Koh, and colleagues in this issue of the JCI, elegant experiments that establish this causal link are described (12). 


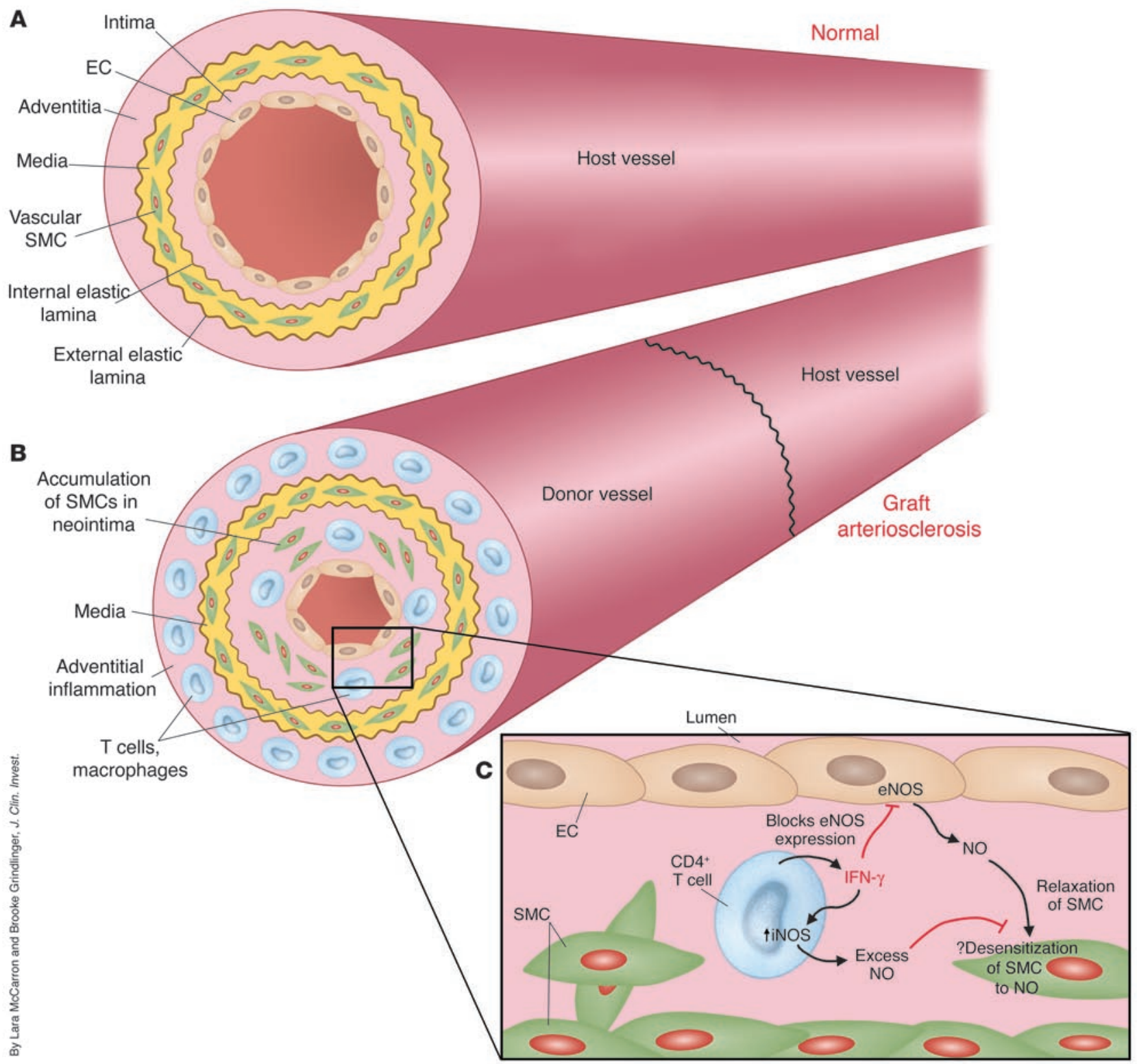

Figure 1

Pathology and mechanisms of graft arterial disease. (A) A cross section of a normal muscular artery is shown. The thin intima includes the endothelial lining of the lumen and minimal subendothelial connective tissue. The media is demarcated by internal and external elastic lamina and includes vascular SMCs. The adventitia is a loose connective tissue sheath outside the media. There are few or no inflammatory cells in any of the layers. (B) In graft arteriosclerosis, inflammatory cells, including T cells and macrophages, infiltrate both the adventitia and the intima. Furthermore, SMCs derived from either the media or circulating host progenitors enter the intima and proliferate. This results in thickening of the lumen and eventual luminal narrowing. (C) A proposed mechanism of intimal SMC proliferation is shown, based on recent data from the Pober laboratory (12). IFN- $\gamma$ is produced by alloreactive T cells within graft arterial walls. The IFN- $\gamma$ blocks the expression of eNOS and enhances the expression of iNOS by the T cells themselves. Excess NO produced by the T cells may desensitize SMCs to the relaxation and antiproliferative effects of NO. Neutralization of IFN- $\gamma$ blocks changes in eNOS and iNOS expression induced by alloreactve T cells, and a pharmacologic inhibitor of iNOS partially blocks development of intimal SMC accumulation.

\section{IFN- $\gamma$ dysregulates graft arterial eNOS and iNOS expression}

Pober and colleagues again turn to the human-to-mouse arterial interposition graft model. They show that 7-9 days after allogeneic PBMC transfer (before intimal thickening can be detected), excised grafts have impaired responses to NO-dependent vasodilators bradykinin and substance $\mathrm{P}$, compared with grafts from mice that did not receive allogeneic PBMCs (12). At that time, SMCs were observed to contract normally in response to prostaglandin $\mathrm{F}_{2 \alpha}$ and relax normally in response to the NO- donor nitroprusside. EC dysfunction persisted for two weeks after PBMC transfer, and at that time, SMC dysfunction was also evidenced by impaired responses to both prostaglandin $\mathrm{F}_{2 \alpha}$ and nitroprusside. These results indicate early vascular graft dysfunction in a model that eventually 
develops intimal hyperplasia. Blocking antibodies were employed to demonstrate that IFN- $\gamma$ is required for PBMC-induced $\mathrm{EC}$ and SMC dysfunction.

The Pober group also shows that EC and SMC dysfunction in the grafts correlates with dysregulated NOS expression (12). Consistent with endothelial dysfunction, eNOS mRNA was reduced, but, unexpectedly, iNOS expression was increased. Immunohistochemical data suggest that iNOS is expressed by infiltrating human T cells. Anti-IFN- $\gamma$ also blocked the PBMC-induced changes in eNOS and iNOS expression. A causal link between iNOS expression and SMC dysfunction was demonstrated using $1400 \mathrm{~W}$, a specific inhibitor of iNOS. This inhibitor partially restored nitroprusside-induced relaxation of dysfunctional grafts when administered ex vivo and partially blocked the development of PBMC-dependent SMC dysfunction when administered in vivo. Importantly, 1400W treatment also blocked graft intimal hyperplasia.

This study moves the field of graft arteriosclerosis forward by establishing a relationship between IFN- $\gamma$, early EC and SMC dysfunction, and intimal hyperplasia (Figure 1C). Many additional interesting questions are raised. Allogeneic responses to graft ECs led to reduced levels of eNOS and elevated levels of iNOS (in T cells); however, it is unclear whether the net amount of NO produced was altered (12). Paradoxically, increased iNOS activity appeared to cause intimal SMC proliferation in the grafts (12) even though NO is known to block SMC proliferation (13), and cardiac allografts in iNOS-deficient mice actually exhibited augmented arteriopathy (14). Whether this contradiction is attributable to species or model differences is not resolved. The possibility that excess NO produced by elevated iNOS may desensitize SMCs to NO in vivo also requires further investigation. Although this study (12) carefully analyzes the downregulation of eNOS by $\mathrm{CD}^{+} \mathrm{T}$ cells in vitro, we do not know if EC dysfunction is necessary for subsequent SMC dysfunction or intimal hyperplasia. Recent data indicate that intimal smooth muscle cell accumulation after mechanical arterial injury is also enhanced by $\mathrm{T}$ lymphocytes (15). In light of the findings of the Pober study, it will be of great interest to examine the INF- $\gamma$-NO link in the setting of mechanical injury as well as in other forms of vascular disease.

Caution is warranted in extrapolating the findings from the arterial graft model to graft arteriopathy in intact organ transplants since alloresponses in the parenchyma may well contribute to the vascular disease. Finally, it is important to acknowledge that IFN- $\gamma$ has many biological effects on the vessel wall. Some are proinflammatory, such as upregulation of endothelial adhesion molecules (5), while others are anti-inflammatory, such as induction of negative regulators of $\mathrm{T}$ cell activation $(16,17)$. In mouse allograft models, the absence of IFN- $\gamma$ significantly enhances acute rejection $(18,19)$. Therefore, therapeutically targeting IFN- $\gamma$ to prevent dysregulated NO production may have unintended consequences.

Address correspondence to: Andrew H. Lichtman, Department of Pathology, Brigham and Women's Hospital, Harvard Medical School, 77 Avenue Louis Pasteur, NRB7, Boston, Massachusetts 02115, USA. Phone: (617) 525-4335; Fax: (617) 525-4333; E-mail: alichtman@rics.bwh.harvard.edu

1. Mitchell, R.N. 2004. Allograft arteriopathy: pathogenesis update. Cardiovasc. Pathol. 13:33-40.

2. Nagano, H., et al. 1997. Interferon-gamma deficiency prevents coronary arteriosclerosis but not myocardial rejection in transplanted mouse hearts. J. Clin. Invest. 100:550-557.

3. Raisanen-Sokolowski, A., Glysing-Jensen, T., Koglin, J., and Russell, M.E. 1998. Reduced transplant arteriosclerosis in murine cardiac allografts placed in interferon-gamma knockout recipients. Am. J. Pathol. 152:359-365.

4. Russell, P.S., Chase, C.M., Winn, H.J., and Colvin, R.B. 1994. Coronary atherosclerosis in transplant- ed mouse hearts. III. Effects of recipient treatment with a monoclonal antibody to interferon-gamma. Transplantation. 57:1367-1371.

5. Choi, J., Enis, D.R., Koh, K.P., Shiao, S.L., and Pober, J.S. 2004. T lymphocyte-endothelial cell interactions. Annu. Rev. Immunol. 22:683-709.

6. Lorber, M.I., et al. 1999. Human allogeneic vascular rejection after arterial transplantation and peripheral lymphoid reconstitution in severe combined immunodeficient mice. Transplantation. 67:897-903.

7. Tellides, G., et al. 2000. Interferon-[gamma] elicits arteriosclerosis in the absence of leukocytes. Nature. 403:207-211.

8. Wang, Y., et al. 2004. Interferon-gamma plays a nonredundant role in mediating $\mathrm{T}$ cell-dependent outward vascular remodeling of allogeneic human coronary arteries. FASEB J. 18:606-608.

9. Liu, Z., et al. 1998. Nitric oxide and endothelin in the development of cardiac allograft vasculopathy. Potential targets for therapeutic interventions. Atherosclerosis. 140:1-14.

10. Behrendt, D., Ganz, P., and Fang, J.C. 2000. Cardiac allograft vasculopathy. Curr. Opin. Cardiol. 15:422-429.

11. Davignon, J., and Ganz, P. 2004. Role of endothelial dysfunction in atherosclerosis. Circulation. 109(Suppl. 1):III-27-III-32.

12. Koh, K.P., et al. 2004. T cell-mediated vascular dysfunction of human allografts results from IFN- $\gamma$ dysregulation of NO synthase. J. Clin. Invest. 114:846-856. doi:10.1172/JCI200421767.

13. Cornwell, T.L., Arnold, E., Boerth, N.J., and Lincoln, T.M. 1994. Inhibition of smooth muscle cell growth by nitric oxide and activation of cAMPdependent protein kinase by cGMP. Am. J. Physiol. 267:C1405-C1413.

14. Koglin, J., Glysing-Jensen, T., Mudgett, J.S., and Russell, M.E. 1998. Exacerbated transplant arteriosclerosis in inducible nitric oxide-deficient mice. Circulation. 97:2059-2065.

15. Boehm, M., et al. 2004. Bone marrow-derived immune cells regulate vascular disease through

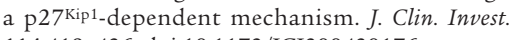
114:419-426. doi:10.1172/JCI200420176.

16. Mazanet, M.M., and Hughes, C.C. 2002. B7-H1 is expressed by human endothelial cells and suppresses $\mathrm{T}$ cell cytokine synthesis. J. Immunol. 169:3581-3588.

17. Rodig, N., et al. 2003. Endothelial expression of PD-L1 and PD-L2 down-regulates CD8+ T cell activation and cytolysis. Eur. J. Immunol. 33:3117-3126.

18. Nagano, H., et al. 1998. Coronary arteriosclerosis after T-cell-mediated injury in transplanted mouse hearts: role of interferon-gamma. Am. J. Pathol. 152:1187-1197.

19. Miura, M., El-Sawy, T., and Fairchild, R.L. 2003. Neutrophils mediate parenchymal tissue necrosis and accelerate the rejection of complete major histocompatibility complex-disparate cardiac allografts in the absence of interferon-gamma. Am. J. Pathol. 162:509-519. 